\section{HYPOTHERMIA; WHAT DOES IT DO TO CEREBRAL OXYGENATION AND MICROCIRCULATION?}

E. Ergenekon, K. Gucuyener, I.M. Hirfanoglu, S. Beken, F. Kulali, E. Koc, E. Onal, C. Turkyilmaz, Y. Atalay

\section{Pediatrics, Gazi University, Besevler,} Ankara, Turkey

Background: Hypothermia is becoming a commonly used treatment for newborns with hypoxic ischemic encephalopathy (HIE) with promising results. The cerebral metabolic effects have been studied extensively.However the acute effects on cerebral and peripheral oxygenation , fractional tissue oxygen extraction, and microcirculation still need further research.

Methods: Newborns with HIE were cooled for 72 hours. Cerebral, peripheral oxygenation index were measured before, during and after hypothermia by near infrared spectroscopy (NIRS), microcirculation was assessed from axilla by sidestream dark field (SDF) imaging technique during and after hypothermia.

Results: We present results of cerebral tissue oxygen index (cTOI) measured by (NIRS) from scalp, calculated cerebral fractional tissue oxygen extraction (cFTOE), microvascular flow index (MFI), $\%$ of vessels with sluggish flow measured by (SDF) technique in 5 newborns with HIE who underwent selective head cooling for 72 hours in figure.
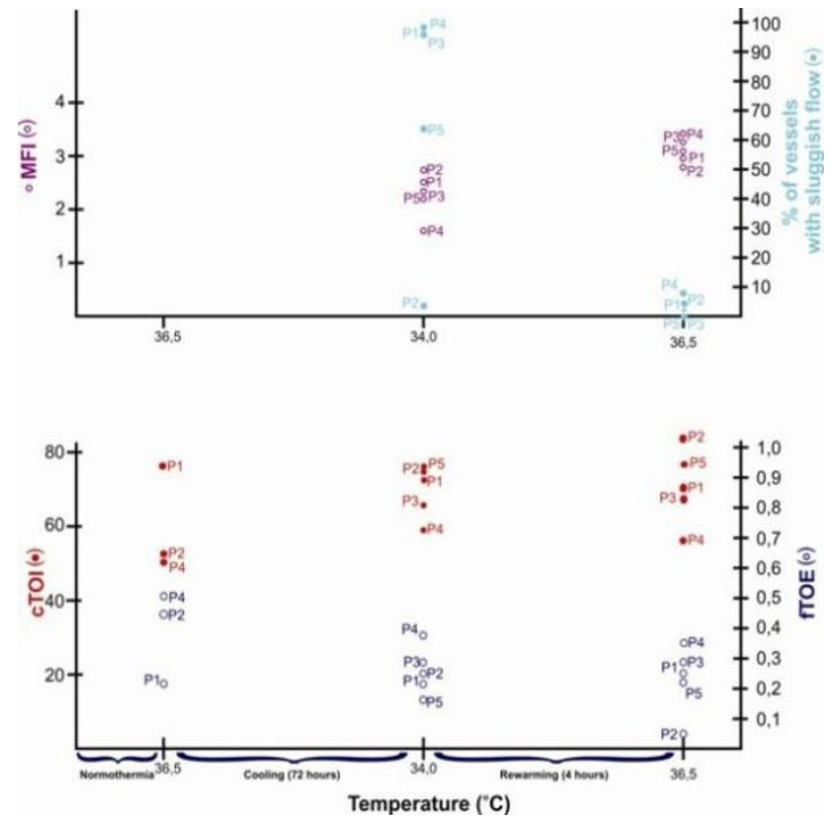

[Microcirculation and NIRS Data During Hypothermia]
cTOI,cFTOE values were similar during hypo and normothermia, however microcirculation was much slower during hypothermia.

Discussion: Selective head cooling causes minor effects on cerebral oxygenation measured by NIRS, however the repercussions of its effects on blood flow velocity in capillaries require further research.

319

\section{EFFECTS OF BLOOD SAMPLING ON CEREBRAL TISSUE OXYGENATION INDEX (TOI) IN VLBW INFANTS: VENEPUNCTURE VERSUS HEEL LANCE}

\author{
N. Hess ${ }^{1}$, B.M. Hüning ${ }^{2}$, C. Roll ${ }^{1}$ \\ ${ }^{1}$ Department of Neonatology and Paediatric \\ Intensive Care, Vest Children's Hospital \\ Datteln, University of Witten-Herdecke, Datteln, \\ ${ }^{2}$ Department for Paediatrics I, University of \\ Duisburg-Essen, Essen, Germany
}

Background and aims: In term neonates venepuncture is considered less painful than heel lance for blood sampling. Here, we compared the effects of venepuncture and heel lance blood sampling in preterm infants $<1500 \mathrm{~g}$ birth weight.

Methods: Twenty infants (median [range] birth weight 855 [355 - 1470] g, gestational age 28 [24 - 32] weeks) were studied during sequentially performed venepuncture and heel lance blood sampling. Heart rate and arterial oxygen saturation were registered continuously. Cerebral tissue oxygenation index (TOI) was measured by near infrared spectroscopy. Cerebral fractional oxygen extraction (FOE) was calculated.

Results: During both procedures, heart rate increased and TOI decreased significantly. The decrease in TOI was significantly larger during venepuncture (from 64 [45-99]\% to 57 [47-95\%]) than during heel lance blood sampling (from 68 [51-99]\% to 63 [47-99] \%, $p=0.05)$. Arterial oxygen saturation decreased and FOE increased during venepuncture only.

Conclusions: Our results indicate that - in contrast to term neonates - for preterm infants venepuncture is more stressful than heel lance blood sampling. 